\title{
Interactive Cognitive Training Tool Designed for Autism Spectrum Disorder Children
}

\author{
Yang Liu, ${ }^{1}$ Shaoping Zuo, ${ }^{2 *}$ and Chun-Liang $\mathrm{Hsu}^{3}$ \\ ${ }^{1}$ School of Electronics and Electrical Engineering, Dongguan Polytechnic, \\ No. 3, University Rd., Song-Shang Lake District, Dongguan, Guangdong 523808, China \\ ${ }^{2}$ Department of Autism, Dongguan Rehabilitation Experimental School, \\ No. 5, Longzhang Rd., Dongcheng District, Dongguan, Guangdong 523808, China \\ ${ }^{3}$ Department of Electronic Engineering, St. John's University, \\ No. 499, Sec. III, Tan-Kin Rd., Tan-Shuei District, New-Taipei City 25135, Taiwan
}

(Received June 30, 2020; accepted December 7, 2020)

Keywords: autism, cognitive training, LED, automatic speech recognition, IR positioning

With the continuous advancement of technology and the development of sensor technology and data science, autism diagnosis technology has developed rapidly, and children with autism can be diagnosed earlier. However, there is no effective treatment so far. Comprehensive rehabilitation can only be implemented through early intervention methods. To improve the effect of early intervention on the cognitive learning of autistic children and overcome the limited focus, lack of interest to small children, and other problems in traditional cognitive learning, we designed an interactive cognitive training tool for cognitive learning of the characteristics of color for autistic children. The training tool uses LED display technology, voice recognition technology, and IR positioning technology to realize the interactive design of color recognition training for autistic children.

\section{Introduction}

Autism spectrum disorder (ASD) is a lifelong neurodevelopmental disorder characterized by persistent difficulties in social communication, social interaction, and the presence of stereotyped or repetitive behaviors. ${ }^{(1)}$ Although the exact causes of ASD are not known and there is no cure, research has shown that intensive, early intervention programs can improve cognitive and language abilities and adaptive behavior in children with ASD. ${ }^{(2)}$ Early screening to identify ASD and provide interventions that are known to produce positive results is essential for autism rehabilitation. ${ }^{(3)}$

In recent years, with the continuous development of computer science and sensor technology, sensor technology has shown great potential for improving the behavioral assessment and intervention for children with $\mathrm{ASD} .{ }^{(4)}$ By collecting specific data, these sensors can obtain specific behavior information of such children. In this way, the processed data from these sensors can then be used to screen children for disorders and plan interventions. ${ }^{(5)}$ Autism screening and intervention technologies based on computers, mobile devices, virtual reality 
systems, and social robots have been widely researched and applied, and have achieved good therapeutic effects. At the same time, there are also problems such as high treatment costs and the need for professional therapist assistance. ${ }^{(6)}$ In addition, owing to the young age of autistic children, there are also problems such as the inability to use computers and virtual reality systems alone, and traditional card teaching is still used. Card teaching can be very effective for high-functioning autistic children, but for low-functioning autistic children with learning difficulties or even mental retardation, the effect is not ideal and its efficiency is extremely low. However, the fusion of sensor technology and machine learning technology has the possibility of solving these problems. We have designed a convenient interactive cognitive training tool based on sensor data collection and machine learning for children with ASD.

The cognitive training tool uses LEDs to display color, graphics, and other cognitive training information, collects and recognizes the words and gestures of trained children through sensor data collection technology, and aims at early childhood color cognitive education, combined with color education for children with autistic cognitive characteristics, using information such as voice response time, gesture operation response time, and accuracy rate as feature information. The tool realizes the recognition and judgment of color in cognitive training through machine learning algorithms, and displays it through the wireless terminal. The specific functions are as follows:

A. A multicolor display of color through an LED screen, including a static display, a dynamic display, changes in lightness and darkness, and a contrast display;

B. While displaying a color, prompt sounds are emitted through the loudspeaker. Then the voice recognition module recognizes the uttered reply, and the IR sensor module recognizes gesture operation, through which interactive autonomous operation learning is realized;

C. The training tool has different training modes, which can be switched through wireless terminals without interference according to the training situation of children.

\section{Related References and System Design Principles}

\subsection{Related references}

In the past few decades, the prevalence of ASD has risen sharply. The education and rehabilitation of autistic children has attracted much attention. The development of technology has provided tremendous help for the early screening and intervention treatment of autistic children. Studies have shown that early comprehensive and targeted behavioral interventions can improve social communication and reduce anxiety and aggressiveness. ${ }^{(7)}$ Cognition of color is a part of early cognitive learning in children, but it is of great significance for future communication learning. However, many autistic children are different from ordinary children in color perception, and have differences in color preference and color discrimination ability. Children with autism often have the characteristics of a preference for cool colors, poor color discrimination ability, and color discrimination related to color preferences. ${ }^{(8,9)}$ The development of technology in recent years, especially sensor technology, computer-aided technology, and robot technology, has helped provide access to educational opportunities and life experiences, 
and has facilitated engagement with knowledge and other people. ${ }^{(10)}$ Assistive technologies combine speech, pictures, words, and animation in interactive ways to structure concepts that suit the level of understanding of learners and their interests. ${ }^{(11)}$ Computer-based teaching and game-based learning can make a major practical contribution to the teaching of basic life skills for people with autism. ${ }^{(12)}$ We can explore the use of sensory interaction technology to enhance the interest of children with autism in color cognitive learning. For example, adding sensory interactions such as sounds and light flashes can attract the attention of autistic children and increase their participation time in related learning activities. ${ }^{(13)}$ Similarly, we can collect various feature information of participating children through sensors, and use machine learning algorithms to identify and make judgments on children's behavior characteristics. ${ }^{(14)}$ LED-based display technology has been widely used. LED-based display screens can achieve multiple levels of brightness and full true-color displays to enhance color effects. Non-specific voice recognition technology can be implemented and operated by interactive commands, realizing vocal interactive communication under specific instructions to meet children's rehabilitation learning needs. ${ }^{(15)}$ At the same time, in order to enhance the fun of the interaction, the auditory, visual, and tactile senses are integrated into the learning and training. Based on interactive IR electronic whiteboard technology, ${ }^{(16)}$ we have realized the positioning of children's gesture operations through IR positioning technology. We designed a cognitive training tool based on this technology for children with autism, as described in this article.

\subsection{Structure design concept}

Our aim was to design a convenient and low-cost acousto-optic interactive cognitive training tool for autistic children, which is suitable for rehabilitation training in special education schools. Through this training tool, teachers or parents can assist children to carry out color cognitive rehabilitation training. The training tool has multiple training modes and can carry out rehabilitation training for children of different levels and ages. As shown in Fig. 1, after selecting the training mode, the device displays color information through the LED display, vocalizes the name of the color while playing music, repeats the prompt, and then asks questions. The voice recognition module is used to recognize children's voices, and the control system obtains voice information and makes judgments, then vocalizes excitation sounds through speakers, and strengthens the display through LEDs. To enhance the training

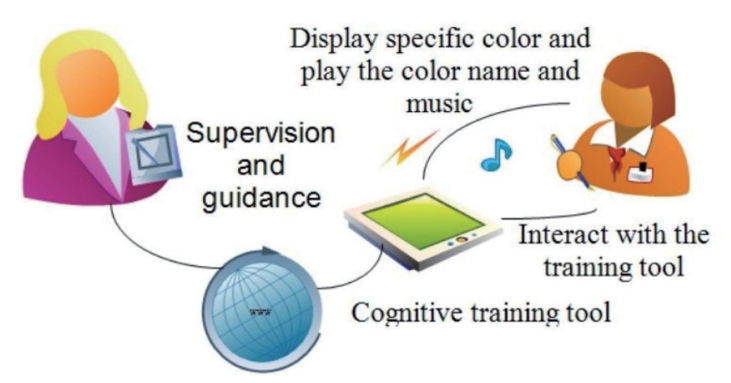

Fig. 1. (Color online) System design concept. 
effect, we added color-matching classification and other modes. Through IR sensor positioning technology, participants can complete interactive operations by touching the LED screen. According to the children's learning situation, the training content can be changed through the mobile terminal to attract their interest.

\section{System Hardware and Software Techniques}

According to the technical literature and design ideas of Sect. 2, we designed the system block diagram shown in Fig. 2. The system realizes interactive color recognition training through the main control system, display module, voice recognition module, IR sensor module, speaker, wireless communication module, and mobile terminal.

\subsection{Display module}

The cognitive training tool for children with autism in this paper is designed to provide assistance in early color cognitive rehabilitation training and enhance the effect of intervention. Children with autism often have problems with color preference and color difference resolution; thus, the use of relatively strong visual stimuli such as colored lights can better attract the attention of participants. Therefore, we choose a $16 \times 16$ LED dot matrix screen for the color display and select a WS2812 dot matrix light source, as shown in Fig. 3. This is an intelligent externally controlled LED light source that integrates a control circuit and a light-emitting circuit. Its appearance is the same as a 5050LED lamp bead, and each element is a pixel. The three primary colors of each pixel can a realize full true-color display with 256 levels of brightness. A circuit diagram for a single WS2812 circuit is shown in Fig. 4(a). When multiple LEDs are connected, as shown in Fig. 4(b), the DOUT pin of the previous LED is connected to the DIN pin of the next LED. Through the dot matrix screen, we can display the color of the entire screen, and also control the blinking rate and dynamic display of individual or regional lamp beads, and attract the attention of participants with music to increase the fun of training and learning.

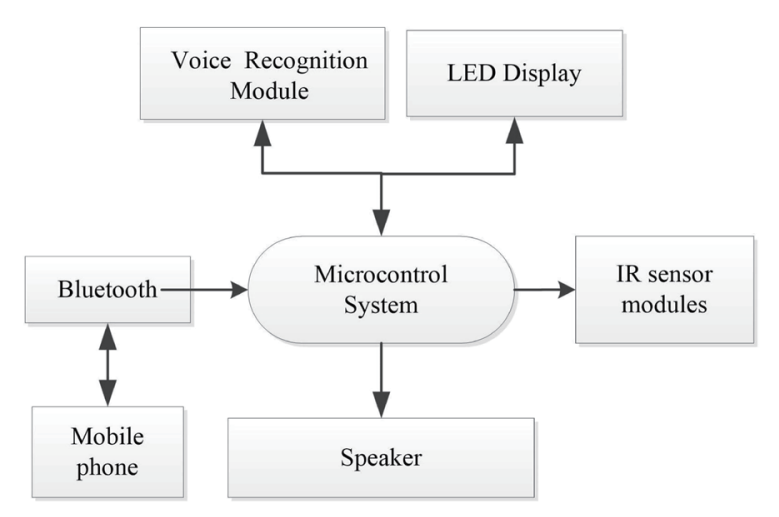

Fig. 2. Block diagram of the training tool.

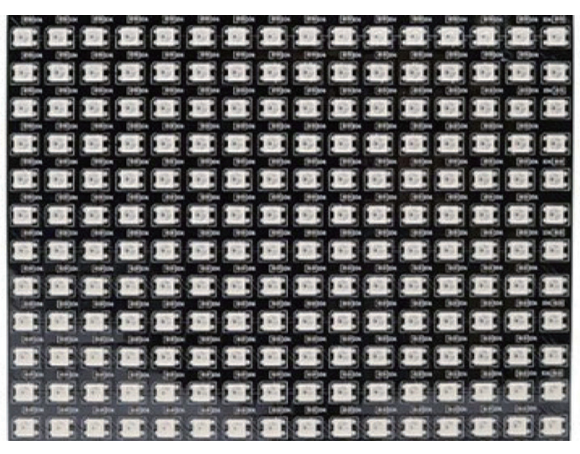

Fig. 3. WS2812 dot matrix screen. 


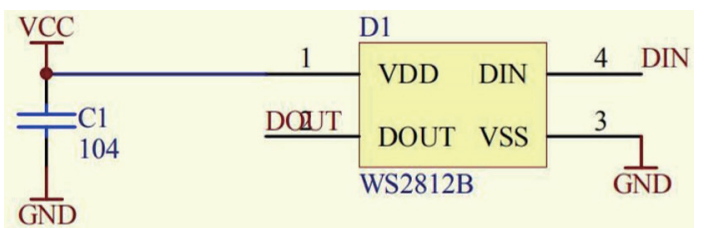

(a)

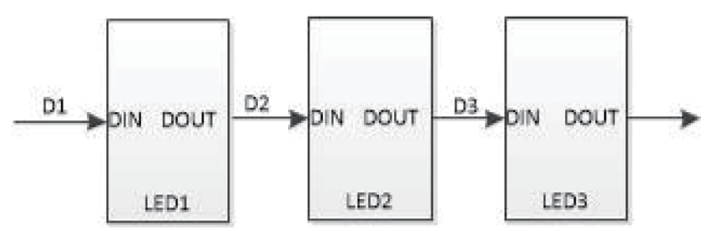

(b)

Fig. 4. (Color online) Circuit connection diagram.

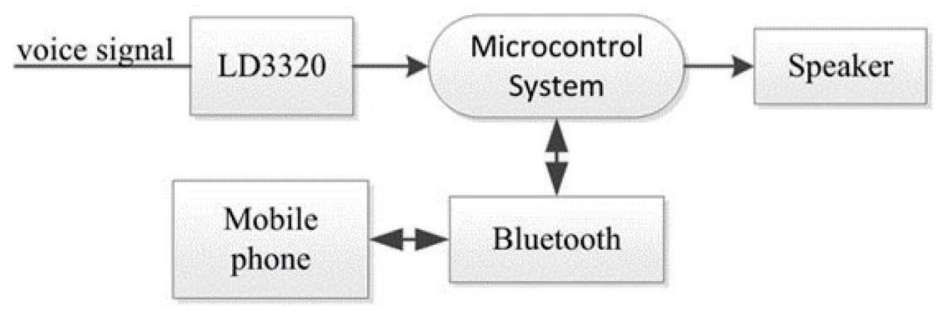

Fig. 5. Block diagram of voice recognition interaction process.

\subsection{Voice interactive recognition}

Voice interactive recognition is input and voices are recognized through a non-specific voice recognition module. Using the internal input Pinyin library, the collected sound signal is converted into Pinyin, which is compared with the input Pinyin. We use an LD3320 chip for the speech recognition module, which is a non-specific voice recognition chip designed and produced by ICRoute. It can recognize up to 50 entries, and each entry can recognize words and phrases with a length not exceeding 10 characters or a 79-byte Pinyin string to meet the needs of this design. As shown in Fig. 5, the voice recognition module recognizes the voice data and sends it to the microcontrol system for processing. The microcontrol system sends the identification information to the mobile terminal via Bluetooth and controls the speaker to respond accordingly.

\subsection{IR recognition and positioning}

An IR sensor emits invisible IR light through an IR emission tube. The IR light is reflected by an obstacle and then received by a receiving tube to detect the obstacle. IR technology has been widely used in interactive electronic whiteboards. Through IR sensor arrays, touch control of electronic whiteboards can be achieved. In order to achieve simple interactive cognitive training and reduce design costs, we partition the display screen, as shown in Fig. 6. We divide the display screen into four positioning points, and each positioning point corresponds to an area. When a certain point is located, all the LEDs in the corresponding area respond interactively. For the positioning of each area, we still use IR sensors, but the IR sensors are different from a traditional IR radiation sensor array. We use the IR sensor module shown in Fig. 7 with an integrated transceiver to achieve positioning. As shown in Fig. 6, the IR sensor 


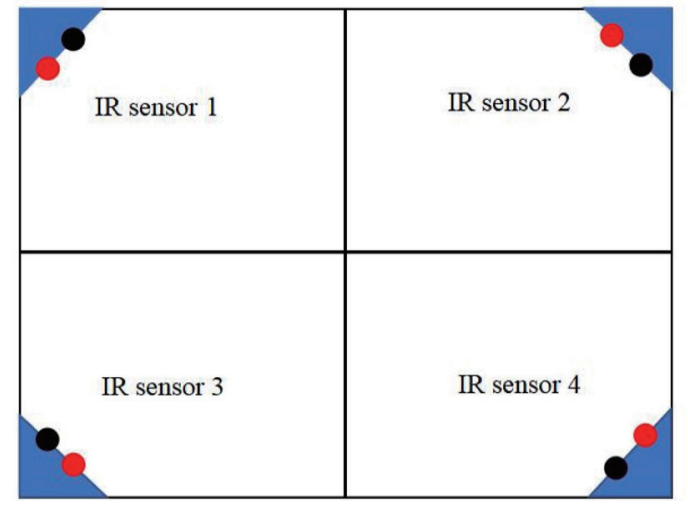

Fig. 6. (Color online) Schematic diagram of IR sensor positioning.

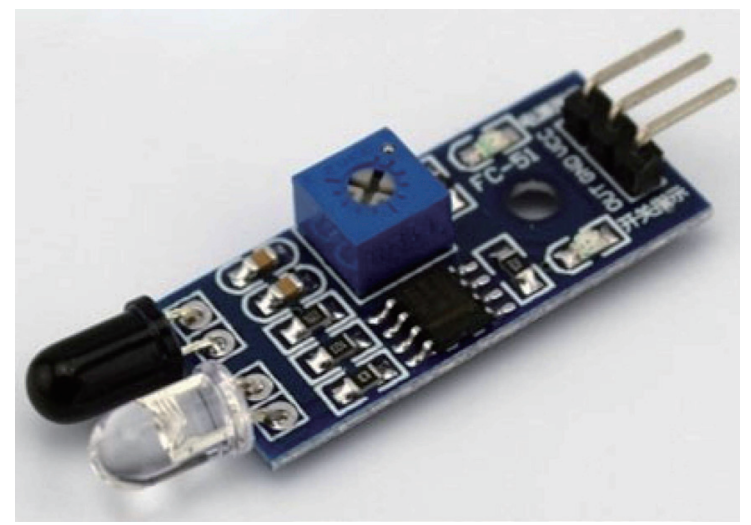

Fig. 7. (Color online) IR sensor module.

module determines whether the participant clicks on the corresponding area by detecting the IR light reflection in the area, thereby achieving positioning.

\section{Results and Discussion}

The interactive training tool for autistic children designed in this paper produces the output used for color intervention training with the LED display and voice prompts, and recognizes children's behavior through IR positioning and voice recognition, thus achieving color rehabilitation training. Compared with the auxiliary tools mentioned in Refs. 10 and 17, we pay more attention to the color training itself, and use the sensor-based interactive technology for color rehabilitation training, to realize intelligent training, and to help teachers and parents carry out rehabilitation training.

\subsection{Display test}

Our cognitive training tool for autistic children is based on acousto-optic interaction. It controls the LED display screen to display different colors or graphics with different intensities through the control system for color cognitive training. Figure 8(a) shows the color full-screen training display; according to the learning situation of the trained children, the LED screen makes different changes in brightness. Figure 8(b) is a multicolor irregular display, which can further improve the color recognition ability of autistic children.

\subsection{Voice interactive recognition}

The system enters the corresponding training instructions according to the training mode, and after booting, it will emit a system prompt tone to guide the participants to carry out color cognitive learning. Participants' responses to questions will affect subsequent learning. The system will make a decision to repeat the learning or enter the next stage of learning based 


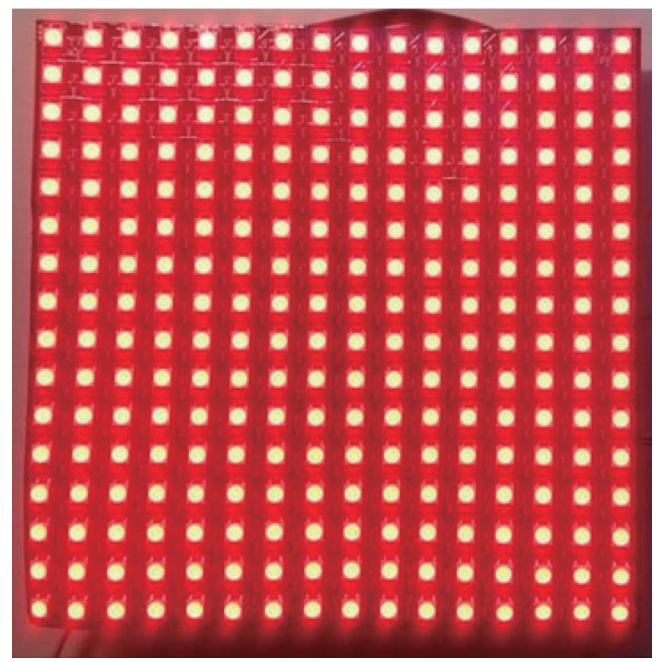

(a)

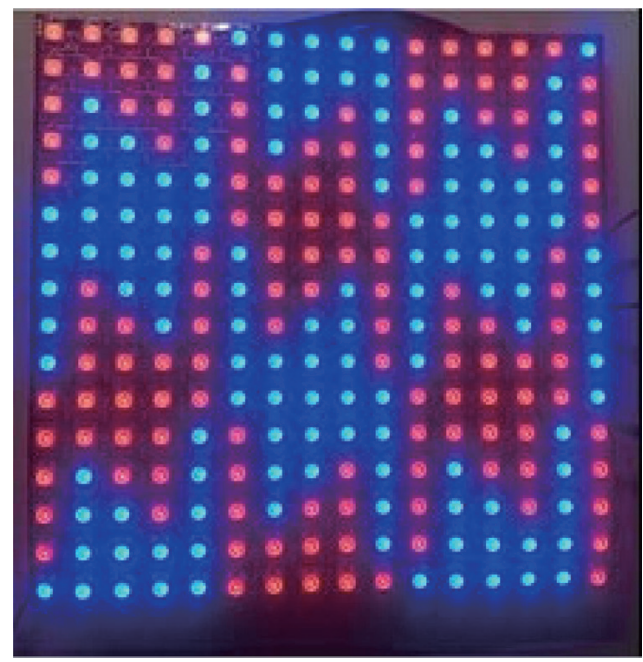

(b)

Fig. 8. (Color online) Color display.

on the voice recognition information. At the same time, the system will also transmit the voice recognition results to the mobile terminal to record information statistics. Because the participating children are young, speech recognition may be affected by pronunciation, etc. This issue needs to be verified by further experiments.

\subsection{Positioning results}

After testing and verification, the IR sensor module can realize children's gesture positioning, and the positioning result is not affected by the displayed light. However, the detection distance and angle of the IR sensor will affect the positioning result, and reducing the detection distance can improve the accuracy of positioning.

\section{Conclusions}

With the development of screening technology, children are being diagnosed with autism at an increasingly young age, and we can carry out intervention treatment earlier. We designed a cognitive training tool for children with autism based on acousto-optic interaction technology to meet the needs of early color cognitive learning for children with autism. The training and learning tool combines the features of autistic children's sensitivity to sound and vision. Through the control of visual and sound stimuli, the basic cognitive interactive training of autistic children is achieved, which overcomes the monotony of traditional color cognitive learning. The cognitive training tool is simple to operate and inexpensive. In follow-up work, we will use this cognitive training tool to carry out experimental verification to further improve its effectiveness for autistic children and provide a reference for practical rehabilitation research on autistic children. 


\section{Acknowledgments}

This work was supported by Young Innovative Talents Projects in Guangdong Province (No. 2018GkQNCX121), the Project for Technical R\&D and Service Team of Dongguan PolytechnicIntelligent Terminal Product R\&D and Technical Service Team (CXTD201802), and the project for the innovative platform in industry and education integration for higher vocational college of guangdong province-the innovative platform in industry and education integration of testing technology for intelligent terminal product (2020CJPT014).

\section{References}

1 A. Cashin, D. A. Sci, and P. Barker: J. Child Adolesc. Psychiatr. Nurs. 22 (2009) 4. https://doi.org/10.1111/ j.1744-6171.2009.00198.x

2 A. M. Daniels and D.S Mandell: Autism Int. J. Res. Pract. 18 (2014) 583. https://doi. org $/ 10.1177 / 1362361313480277$

3 J. DiPietro, A. Kelemen, Y. L. Liang, and C. Sik-Lanyi: Med. 55 (2019) 440. https://doi.org/10.3390/ medicina55080440

4 E. K. Andrea and R. S. Stefan: Sens. 19 (2019) 4787. https://doi.org/10.3390/s19214787

5 J. J. Cabibihan, H. Javed, M. Aldosari, T. W. Frazier, and H. Elbashir: Sens. 17 (2017) 46. https://doi. org/10.3390/s17010046

6 X. Y. Liu, Q. Wu, W. B. Zhao, and X. Luo: Appl. Sci. 7 (2017) 1051. https://doi.org/10.3390/app7101051

7 K. Valencia, C. Rusu, D. Quiñones, and E. Jamet: Sens. 19 (2019) 4485. https://doi.org/10.3390/s19204485

8 S. H. Cao, W. Fei, and A. J. Sun: Chin. J. Special Edu. 5 (2012) 143.

9 N. Yang: The Relationship between Color Preference and Chromatic Discrimination in Autism Spectrum Disorder, Master Thesis, Gui Zhou Normal University (2015).

10 J. Bharatharaj, L. Huang, R. E. Mohan, A. Al-Jumail, and C. Krägeloh: Rob. 6 (2017) 4. https://doi.org/10.3390/ robotics6010004

11 D. J. Brown, D. McHugh, P. Standen, L. Evett, N. Shopland, and S. Battersby: Comput. Edu. 56 (2011) 11. https://doi.org/10.1016/j.compedu.2010.04.014

12 C. Sik Lanyi, D. Brown, P. Standen, J. Lewis, and V. Butkute: Acta Polytech. Hung. 9 (2012) 225.

13 L. L. Song: J. Modern Special Edu. (Higher Education) 8 (2017) 36.

14 Y. Gu: Data Collection and Preliminary Recognition of Autism Spectrum Disorder Early Signs in Children's Toy Playing, Master Thesis, South China University of Technology (2017).

15 Z. L. Lu, X. L. Shen, J. Liu, G. D. Zhang, and H. L. Sun: Chin. High Tech. Lett. 27 (2017) 277. https://doi. org/10.3772/j.issn.1002-0470.2017.03.011

16 Z. Hu: Location Algorithm and Implementation Technology based on Interactive Infrared Whiteboard, Master Thesis, Chongqing University (2018).

17 Y. Dai: Design and Implementation of Auxiliary Teaching Tools in Autism Training Class, Master Thesis, Huazhong University of Science \& Technology (2019).

\section{About the Authors}

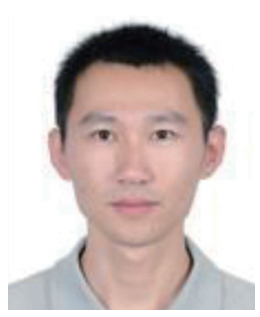

Yang Liu received his B.S. and M.S. degrees in electronic engineering from Northwestern Polytechnical University, Xi'an, China, in 2010 and 2013, respectively. Since 2013, he has worked at Dongguan Polytechnic. His major fields are microprocessor applications, wireless communication, and sensor applications. (liaofan810@126.com) 


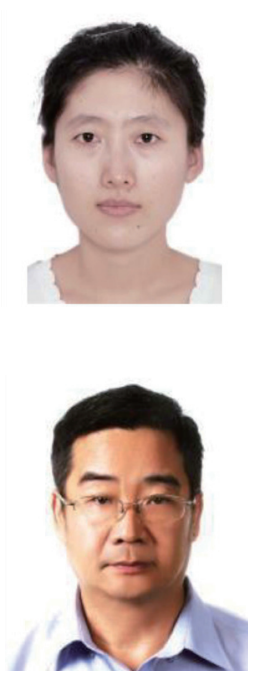

Shaoping Zuo received her B.S. degree in special education from Liaoning Normal University, Da Lian, China, in 2014. Since 2014, she has worked at Dongguan Rehabilitation Experimental School. Her main field is special education. (shaoping_z@126.com)

Chun-Liang Hsu received his B.S. degree from National Taiwan Normal University, Taiwan, in 1977 and his M.S. and Ph.D. degrees from the Industry Education Institute, Taiwan, in 1980 and 2000, respectively. From 2000 to 2009, he was an assistant professor at St. John's University, Taiwan. Since 2010, he has been a professor at St. John's University. His research interests are in microsystem technology, embedded systems, wireless communication, and sensors. (liang@mail.sju.edu.tw; xucl@dgpt.edu.cn) 